Session 3433

\title{
Utilizing Advanced Software Tools for Classroom Projects in Control and Power System Studies
}

\author{
Fanis Chalkiadakis, Recayi Pecen \\ Electrical and Information Engineering Technology \\ Department of Industrial Technology \\ The University of Northern Iowa, Cedar Falls, Iowa
}

\section{Introduction}

Studies in control and electric power systems have always been a major part of the core course requirements of every electrical engineering and/or electrical engineering technology program. In recent years however, undergraduate and graduate student interest has seen a documented decline in both of these areas [1]. This is mainly due to two reasons: a) the abundance of available positions in the computer engineering and the telecommunications industries, and $b$ ) the false impression that electric power and control theory are considered to be "old knowledge" with no space for future development compared to the impressive achievements in the modern fields of computer engineering and telecommunications.

Despite this situation there are still many engineering and engineering technology programs nationwide that offer courses with updated material in the fields of control and electric power systems. The Electrical and Information Engineering Technology (EIET) program at the University of Northern Iowa is one of these programs. It is also the first and only program in the state of Iowa that grants its students with a bachelor's degree in electrical engineering technology after the completion of a four-year course of studies. The EIET program was updated from the Electromechanical Systems (EMS) program in the fall semester of the year 2002.

Recently the program completed its first year of operation with great success and increased enrollment. Part of this achievement is due to the fact that the faculty uses advanced software tools to educate the students that choose the EIET major. These tools are also used by many professionals and utility companies nationwide. Hence, the students are given the opportunity to familiarize themselves with the operation of software packages that most likely they will have to use after they join the workforce. In that spirit, the Department of Industrial Technology has supported the efforts of the EIET faculty for curriculum development with significant classroom renovations, installation of new multimedia equipment and purchase of laboratory apparatus, including hardware and software tools.

Due to the intensity of the program, a substantial portion of the classroom projects that require the use of advanced software is assigned mostly to senior and graduate students in the form of independent studies, and/or final projects. In addition the authors use frequently these software packages for demonstrations during regular class lectures in the courses of Introduction to 
Control Systems (ICS), Industrial Applications of Power Electronics (IAPE), and Advanced Electrical Power Systems (AEPS).

\section{Control and Power System Studies}

Traditionally, studies in control systems require a decent background in mathematics. This has always placed a heavy load of studying time in every student who is enrolled in a control theory class, even when the material is introductory. The ICS is no exception to that rule and this is the reason that the employment of an advanced software package was deemed necessary. While the conventional methods of determining the stability of a control system without the aid of a computer are still taught in that class, the EIET faculty decided to farther train the students by using MATLAB, a simulation software package distributed by Math-Works [2].

The training involves only one introductory session to allow the students to become familiar with the operation of the software and its basic statements and commands that the instructor considers appropriate for future class use. After the first session, solved problems are presented in the class and explanations of the logic and the development of the pertinent code follow. The next step involves the assignment of problems similar to the ones that were solved in the class in order to help the students develop their skills in a progressive manner. Finally, complex problems are assigned that most of the time require the development of separate code listings in order to be fully solved. Occasionally, the verification of a solution with a second method is requested so that the students can become aware of the flexibility and the vast selection of tools that MATLAB offers to the user. This in turn allows them to understand how all these different methods of solving problems can be incorporated into the solution and how they can use a number of them to verify their results and compare them to the ones that they have received by employing the conventional solution methods.

The teaching of the IAPE and AEPS classes follows a similar route with a different software package, the PSCAD/EMTDC distributed by the Manitoba HVDC Research Center in Canada [3]. This real-time simulation software offers a very innovative user interface and an extended library of components ranging from simple passive elements to 3-phase inverter blocks, electric machines and transmission lines. Due to its real-time capabilities, faults and other disturbances can be simulated with virtual "buttons" and "switches" that can open and close at will. It also permits the user to built his own components, including blocks like counters, registers and digital signal comparators. In the background this software package employs state equations and a FORTRAN compiler in order to solve circuits from a few nodes to hundreds of nodes.

In contrast to the ICS course, the use of the PSCAD software package is introduced earlier in the semester. This allows the class to familiarize with the solution of simple circuits that eventually become more complex. It has been observed that the students find the relatively difficult material of the IAPE and AEPS classes more appealing when they use this simulation tool, since it allows them to better understand and actually observe how a circuit functions. In addition the instructors have the ability to offer demonstrations of special setups and system configurations that would otherwise be impossible to perform, considering the extremely high cost of a real life system that can be simulated with a relatively inexpensive software tool. 


\section{Case Studies}

Most independent study projects assigned to senior and/or graduate students in ICS require the use of the MATLAB software package. One representative case study involving the testing of a proportional-integral (PI) control system for stability is presented and the results are discussed. Next, the study of a novel distribution-level compensator is presented. The PSCAD/EMTCD real-time simulation software package is used in this case, and the results of the simulation are also discussed. Similar projects are assigned to graduate students in IAPE and AEPS, while undergraduate students perform studies of lower complexity as part of their homework assignments.

\subsection{Control System Stability Study}

Figure 1 shows a control system in a typical closed-loop configuration [4], which allows for the continuous correction of the output signal, $\mathrm{Y}(\mathrm{s})$. The system consists of four blocks: a) an error detector that calculates the difference between the reference signal $\mathrm{R}(\mathrm{s})$ and the measurement (or feedback) B(s), b) a PI controller that acts in a way that will minimize any errors, c) a plant that translates the input from the controller to a signal that will correct the output, and d) the feedback block that transfers measurements of the controlled variable back to the system in order to perform the closed-loop operation.

The transfer function of the PI controller is given by,

$$
G_{1}(s)=10+\frac{30}{s}
$$

where the gain of the proportional component is 10 and the gain of the integral component is 30 , respectively. In a similar way the transfer function of the plant or process block is given by,

$$
G_{2}(s)=\frac{1}{s+1}
$$

and that of the feedback block is given by,

$$
H_{1}(s)=\frac{1}{s+2}
$$

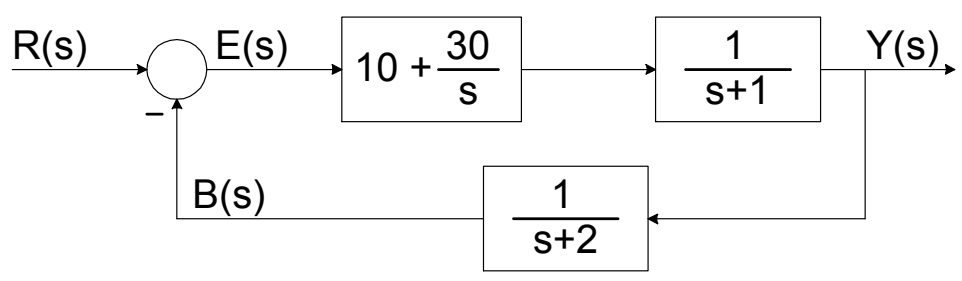

Figure 1. PI control system. 
In order to examine the stability of the system a study of the Bode plots of the open-loop transfer function (OLTF) is required. For this system the OLTF can be written as,

$$
G(s) H(s)=G_{1}(s) G_{2}(s) H_{1}(s)
$$

and after substituting with the equivalent transfer functions it simplifies to,

$$
G(s) H(s)=\frac{10 s+30}{s(s+1)(s+2)}
$$

The plotting of the amplitude and phase values of this function with respect to the frequency can be carried out by means of the MATLAB software package, and the respective code is shown below along with explanatory comments for the function of each line. Similar code is developed for homework and project assignments by the students that enroll in the ICS course [5].

$\mathrm{W}=0.01: 0.1: 1000$;

$\mathrm{f}=(10 * \mathrm{j} * \mathrm{w}+30) \cdot /\left((\mathrm{j} * \mathrm{w}) \cdot{ }^{*}(\mathrm{j} * \mathrm{w}+1) \cdot{ }^{*}(\mathrm{j} * \mathrm{w}+2)\right)$;

$\mathrm{A}=20 . * \log (\operatorname{abs}(\mathrm{f}))$;

$\mathrm{T}=180$ * $^{*}$ angle(f)./pi;

$\operatorname{subplot}(2,1,1) ; \operatorname{semilogx}(\mathrm{w}, \mathrm{A})$

grid on

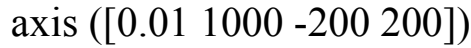

xlabel('Frequency in $\mathrm{rad} / \mathrm{s}$ ')

ylabel('Amplitude in $\mathrm{dB}$ ')

$\operatorname{subplot}(2,1,2) ; \operatorname{semilogx}(w, T)$

grid on

axis ([0.01 $1000-180-60])$

xlabel('Frequency in $\mathrm{rad} / \mathrm{s}$ ')

ylabel('Phase in degrees')
$\%$ Frequency from 0.01 to 1000 , step 0.1

$\%$ Amplitude function

$\%$ Logarithmic value of amp. function

$\%$ Phase function

$\%$ Plot area control (upper part of graph)

$\%$ Grid is on

$\% \mathrm{X}$-Y axes values (amplitude)

$\% \mathrm{X}$-axis label (angular frequency)

$\%$ Y-axis label

$\%$ Plot area control (lower part of graph)

$\%$ Grid is on

$\% \mathrm{X}-\mathrm{Y}$ axes values (phase angle)

$\% \mathrm{X}$-axis label (angular frequency)

$\%$ Y-axis label

The results of this simulation are shown in Figure 2 where it is observed that at the point where the phase angle reaches -180 degrees the amplitude of the open-loop function is negative. This indicates that the control system is stable. With these results as a guide three more studies can be contacted as follows: a) the proportional gain $(\mathrm{P})$ is increased by a factor of 10 while the integral gain (I) stays the same, b) both gains are increased by a factor of 10, and c) both gains are increased by a factor of 20 .

An increase of the proportional gain makes the system more stable as it can be observed in Figure 3. The amplitude of the open-loop transfer function is more negative at a phase angle of -180 degrees than it was before. Changes occur however, when both gains are increased by the same factor. This is evident in Figure 4, where the amplitude is almost zero at a phase angle of -180 degrees, indicating possible stability problems. The area of $-6 \mathrm{~dB}$ is known as marginal stability area and this is where the control system is driven to when both gains are increased [4]. Finally the gain is increased further for the $\mathrm{P}$ and I components of the controller block. The control system is driven to instability, and this is obvious in Figure 5 where the amplitude of the open-loop transfer function is positive for a phase angle of -180 degrees. 

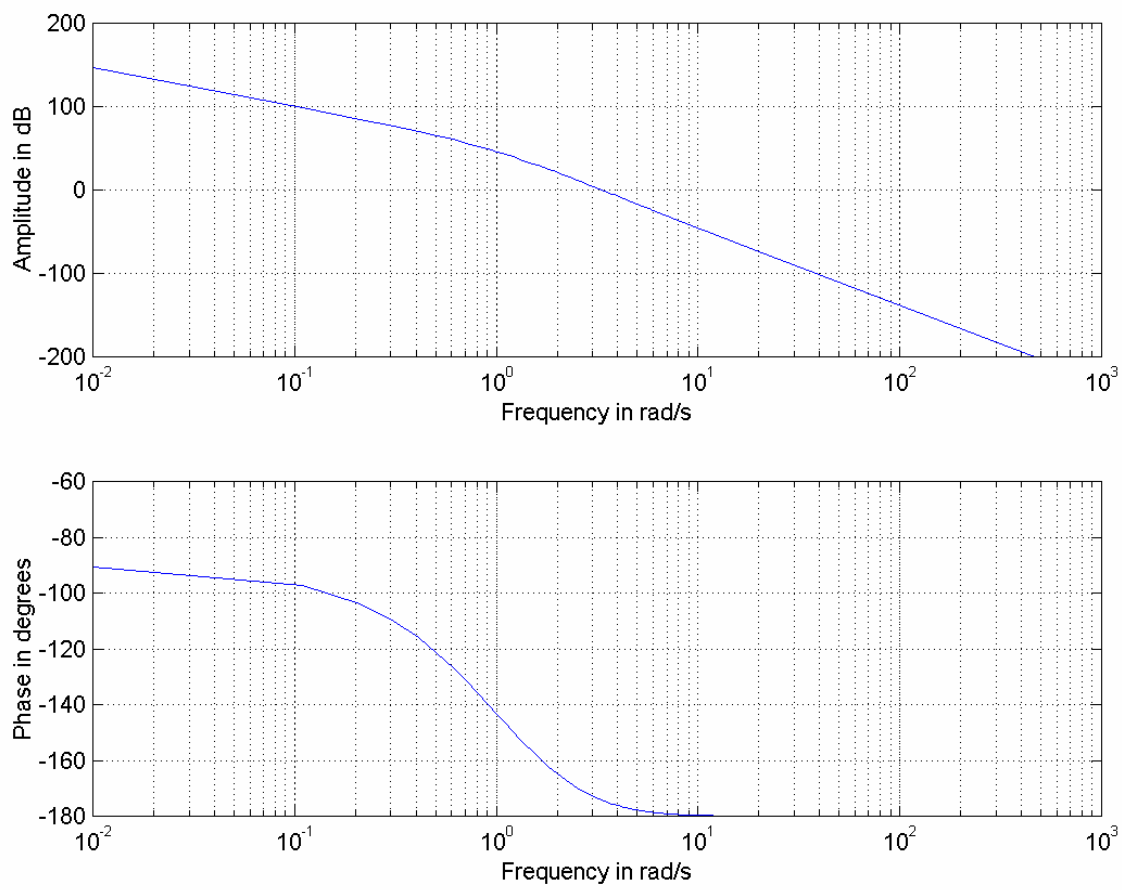

Figure 2. Bode plots for P-gain of 10 and I-gain of 30.
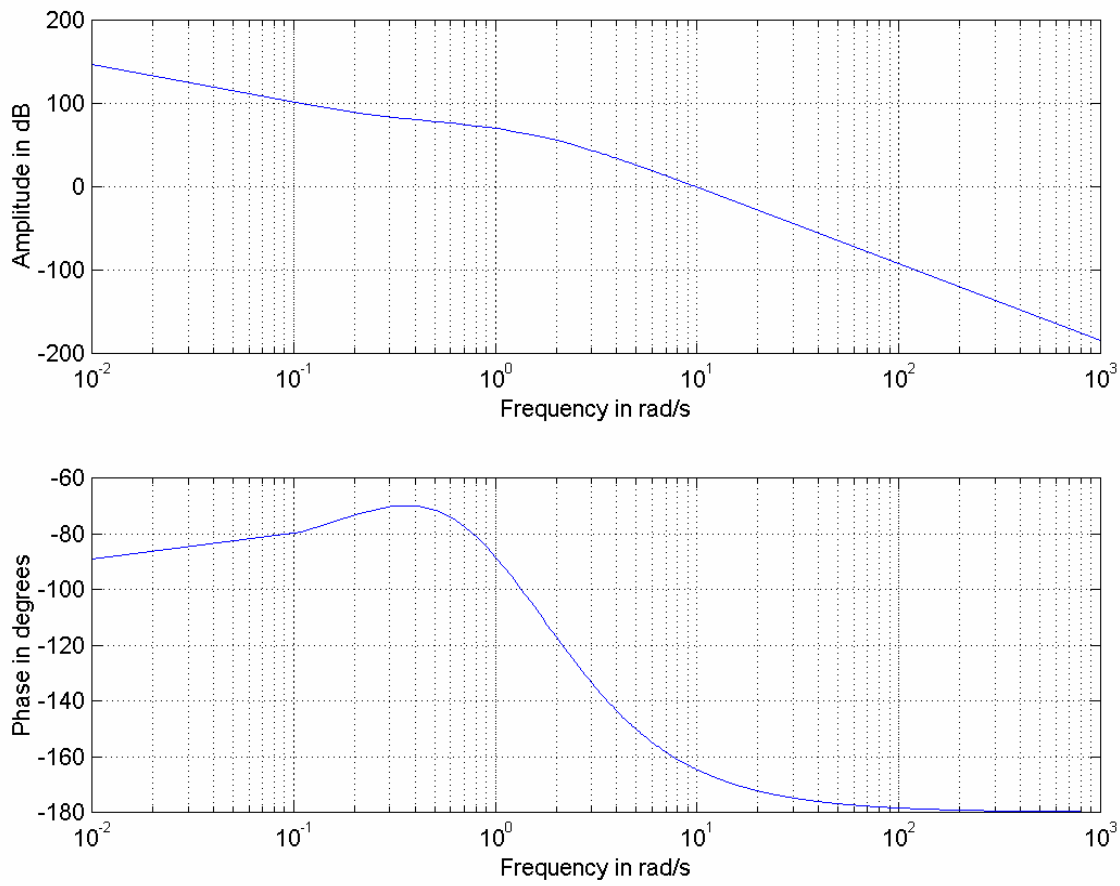

Figure 3. Bode plots with P-gain increased by a factor of 10 . 

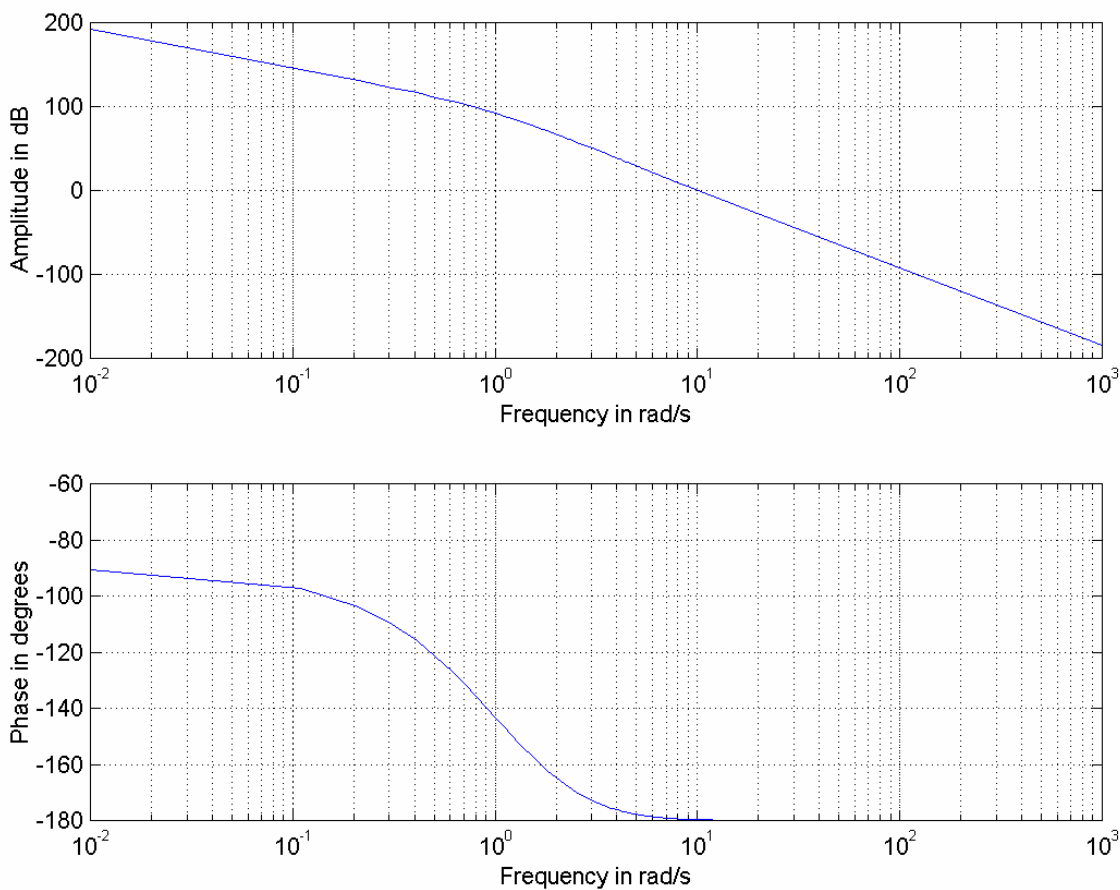

Figure 4. Bode plots for P and I gains increased by a factor of 10 .
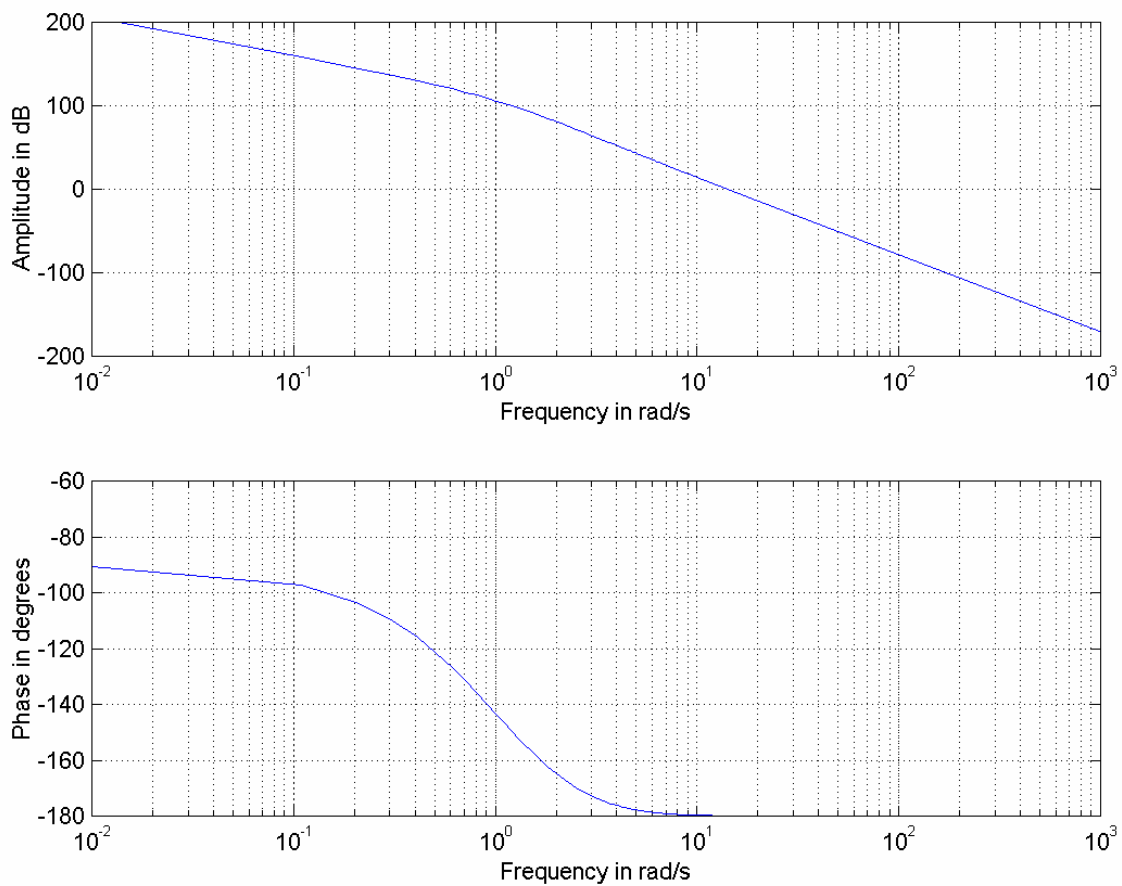

Figure 5. Bode plots for P and I gains increased by a factor of 20 .

Proceedings of the 2004 American Society for Engineering Education Annual Conference \& Exposition Copyright (C) 2004, American Society for Engineering Education 
In order to confirm these results a study in the time domain should be completed. By means of the inverse Laplace transform of the OLTF that MATLAB provides, the response of the control system can be verified. Figure 6 shows its time response for a unit-step input. The second curve clearly illustrates that the P-gain increase improves the behavior of the system in contrast to the first curve. However, instability with oscillations occurs when both gains are increased beyond a certain value and the last curve shows that the system takes a longer time to reach steady state, while maintaining the same value of steady state error in every case.
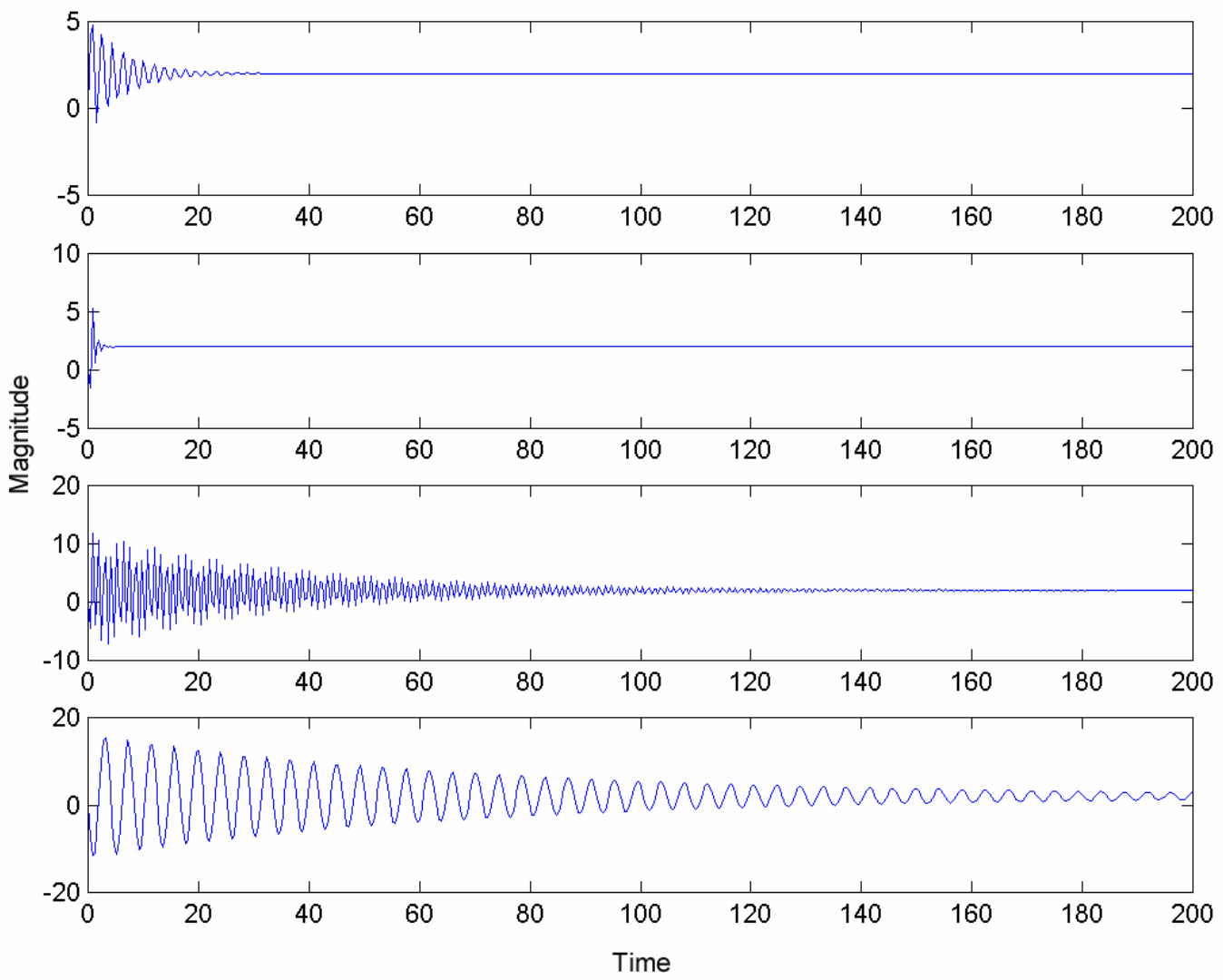

Figure 6. Time response of the PI controller for a unit-step input.

\subsection{Distribution-level Compensator Study}

A novel GTO-based Static Synchronous Compensators (SSC) suitable for distribution level applications is shown in Figure 7 [6]. It consists of two three-level inverters combined in parallel by means of a current sharing inductor, which allows for an equal distribution of the output current. One of the main advantages of this configuration is that it can be connected directly to the network without the use of a three-phase transformer, which is always needed in other GTObased converters for distribution level applications [6].

The valves of the first pole operate as follows: initially valves (1') and (2) turn on and connect point $(\mathrm{P})$ to the neutral. The output voltage is zero. Then valves (1) and (2) turn-on at $\alpha_{1}$ degrees and the output is equal to one half of the dc side voltage. The next step is to turn-on valves ( $\left.1^{\prime}\right)$ and (2) for zero output. Finally by turning-on valves (1') and (2') a negative output is generated at 
point $(\mathrm{P})$ with a magnitude of $\mathrm{V}_{\mathrm{d}} / 2$. From that point on the circle repeats itself. Each step requires that two valves be in the "on" state always $[7,8]$.

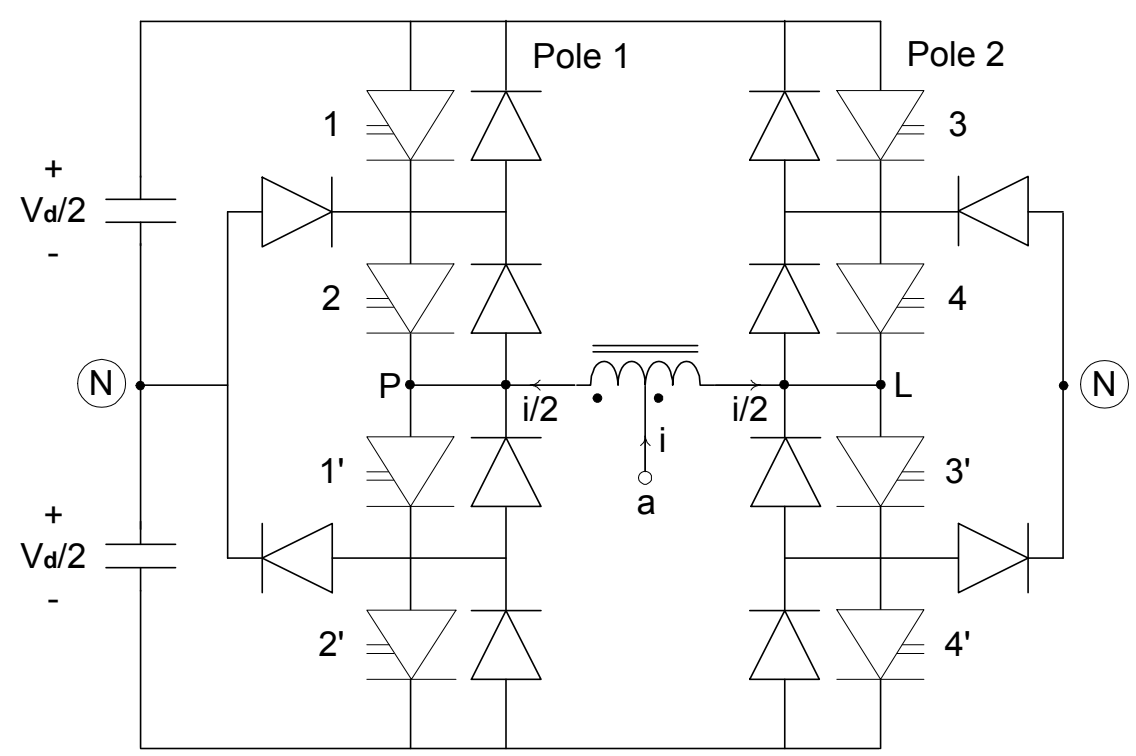

Figure 7. A novel 12-pulse compensator.

The second pole operates in similar way and a different firing angle $\alpha_{2}$. The firing sequence for a three-level output is $\left(3^{\prime}, 4\right),(3,4),\left(3^{\prime}, 4\right),\left(3^{\prime}, 4^{\prime}\right)$. This generates an output voltage of magnitude $\mathrm{V}_{\mathrm{d}} / 2$ at point (L) with reference to the dc neutral. Its rms value is lower than that of the first pole due to the longer delay imposed by the firing angle $\alpha_{2}$.

For a three-phase implementation we use a total of six poles, two per phase. Each pole operates as illustrated in Figures 8(a) and 8(b). With reference to the dc neutral point $(\mathrm{N})$, each pole generates an output voltage with a maximum magnitude of $V_{d} / 2$, where $V_{d}$ is the total voltage of the two capacitors on the dc side. The two outputs (P) and (L), provide only three levels of voltage. Combined however through the current sharing inductor, the output waveform consists of five levels, as shown in Figure 8(c). The line-to-line voltage is shown in Figure 8(d). From this diagram it is obvious that the compensator can provide an output similar to the one of a conventional 12-pulse inverter.

\subsection{PSCAD/EMTDC Simulations}

The performance of this novel compensator is tested with a series of simulations under full load operation, partial load rejection and system fault conditions. The PSCAD/EMTDC software package was chosen because many power utilities and research companies in the power industry are using it and because it has been established as a standard in the power industry.

A block diagram of the simulated system is shown in Figure 9. The SSC is rated at \pm 8 MVAr and it is supported by additional capacitors rated at 3 MVAr. The Short Circuit Capacity (SCC) of the $13.8 \mathrm{kV}$ bus was set at the typical value of $80 \mathrm{MVA}$ with an inductive load connected to the 

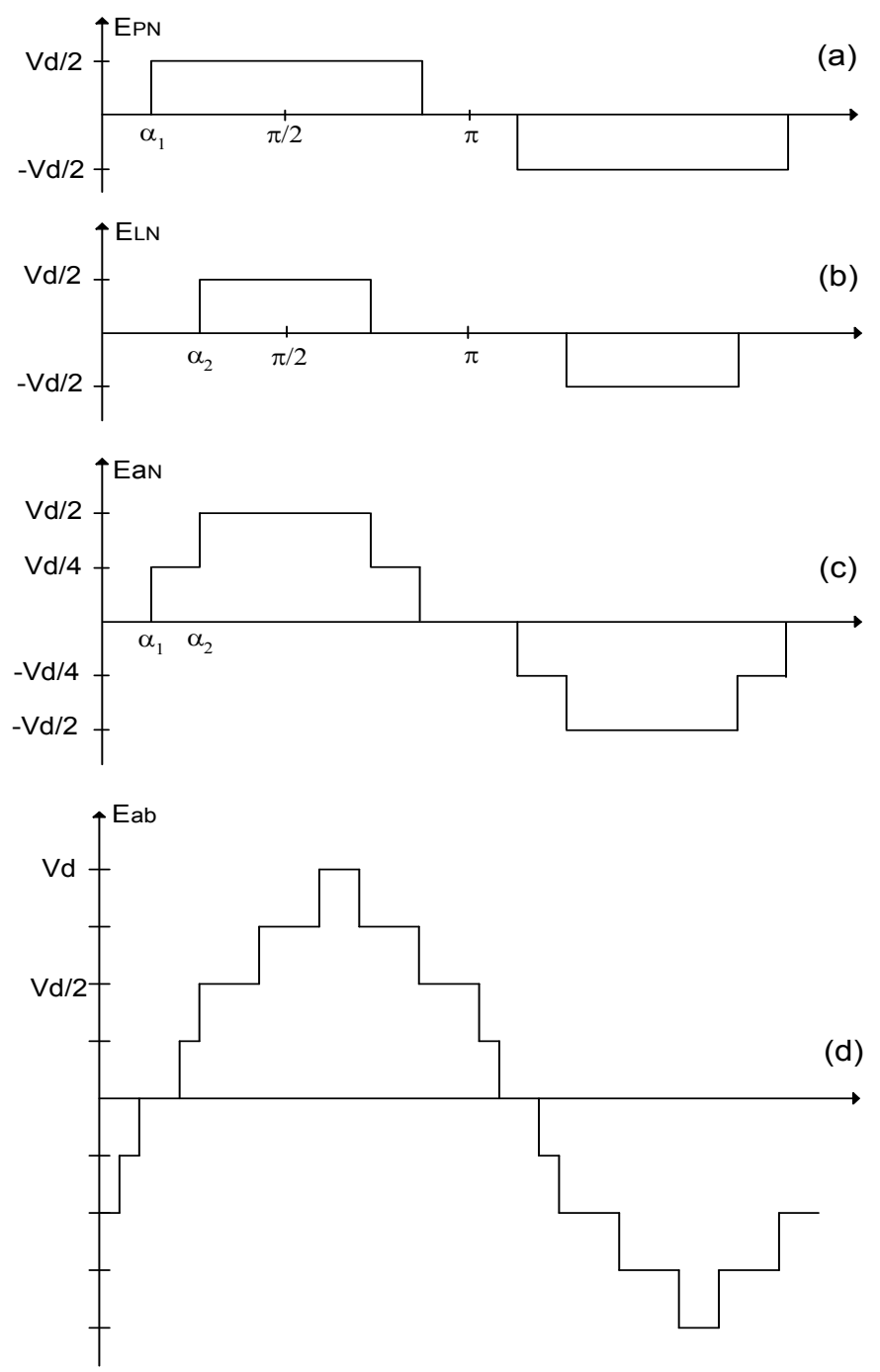

Figure 8. Output waveforms of the compensator.

bus and rated at $30 \mathrm{MW}$ and $10 \mathrm{MVAr}$. The SSC is connected directly to the bus without using a phase-shifting transformer, and when it operates at full load, it produces 7.5 MVAr in order to maintain the load voltage at its rated value. Figure 10 shows the PSCAD simulation diagram.

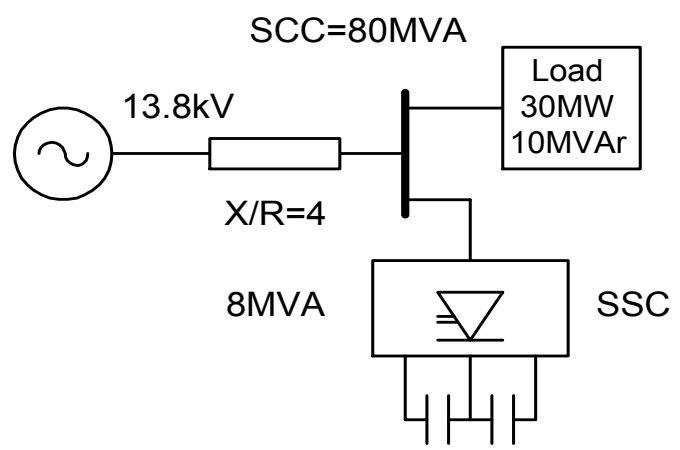

Figure 9. The simulated system. 


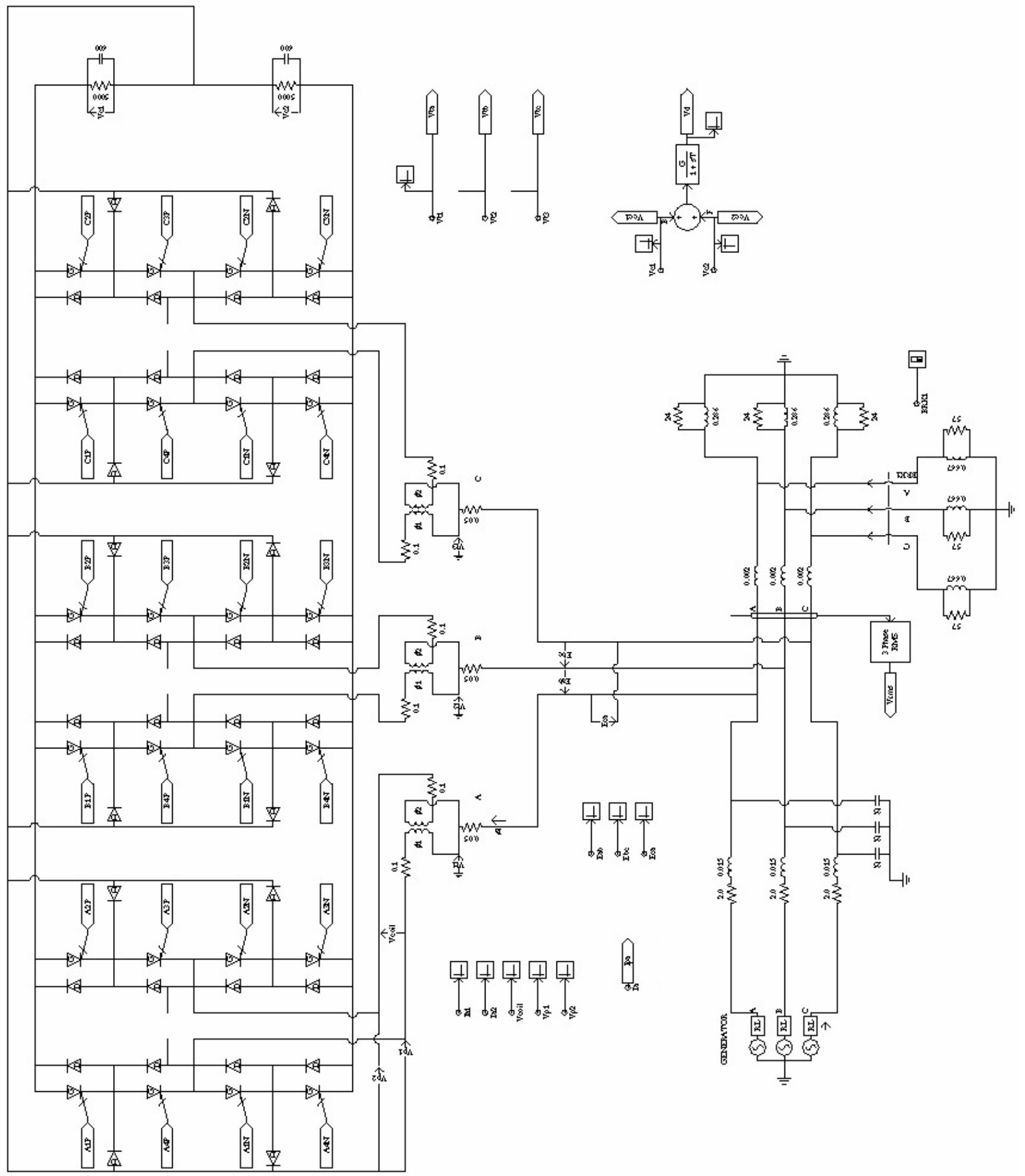

Figure 10. PSCAD simulation diagram of the GTO-based SSC.

Simulation results of the SSC under steady state conditions are shown in Figure 11. A quick observation reveals a slight discrepancy between the theoretical waveforms of the SSC shown in Figure 8, and the simulated results. The steps of the line-to-neutral voltage are shown in Figure 11(a) while those of the line-to-line voltage are shown in Figure 11(b) and they can be easily 
distinguished from each other. The output waveforms on these figures are smoother than those in Figure 8 due to the filtering that is provided by the inductance of the current sharing inductor.

(a)

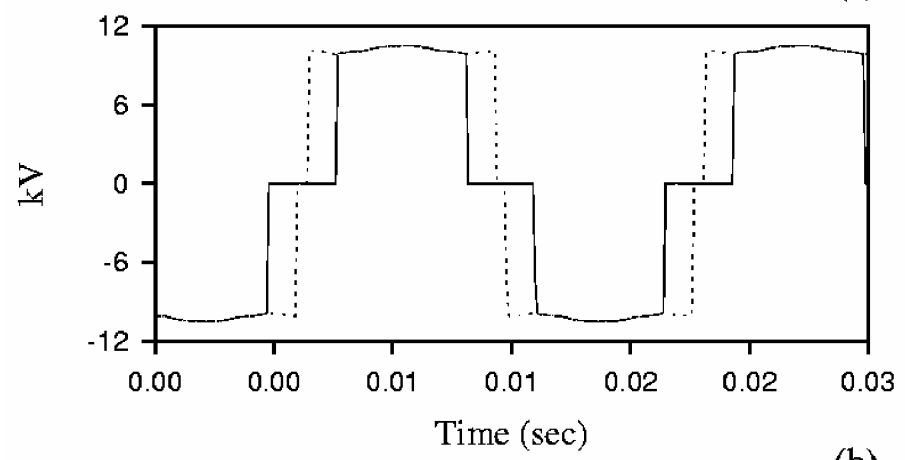

(b)

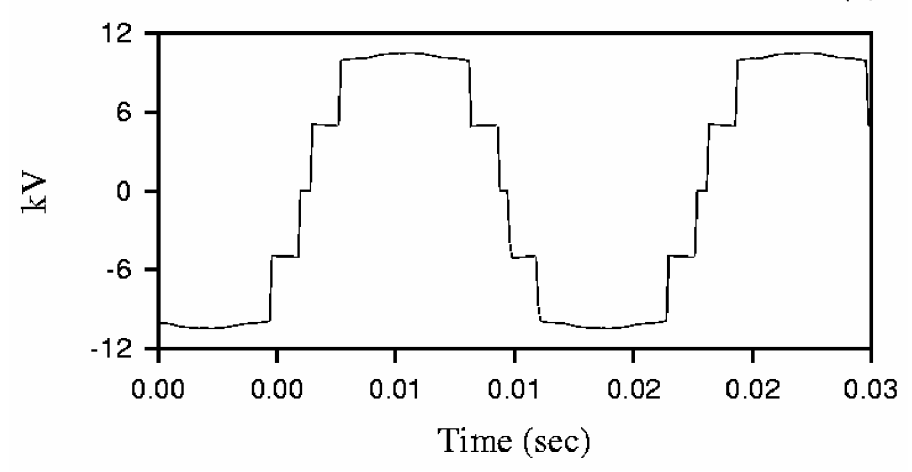

Figure 11. Steady state waveforms.

A load rejection is simulated by opening one of the breakers that connect the load to the power network, at $\mathrm{t}=50 \mathrm{~ms}$ and while the system operates in steady state, shedding $30 \%$ of the load from the bus. The response of the compensator is shown in Figure 12(a). The bus voltage increases due to the reactive imbalance of the rejected load. When this happens, the line voltage regulation loop responds by decreasing the average dc voltage as shown in Figure 12(b). Within 5 cycles after the load rejection occurs, the bus voltage is restored to its rated rms value, indicating the fast response of this control loop.

An important observation is made by examining Figure 12(b). Throughout the transient response of the compensator the two capacitors are maintained equally charged due to the fast and stable response of the equalization control under the changing conditions of the line voltage. Even at the onset of the disturbance the average voltage of both capacitors is the same. This is critical for the stable operation of the SSC. In case of unequal capacitor voltages during a transient period, the second harmonic is introduced to the system [7] and problems may arise concerning the normal operation of the synchronization loop.

Proper operation of the compensator under special conditions, such as an internal fault in the $13.8 \mathrm{kV}$ system is always desirable. Hence a simulated study is performed in order to test the behavior of the compensator. A fault occurs at $\mathrm{t}=50 \mathrm{~ms}$ and while the system operates in steady state. The fault lasts for $100 \mathrm{~ms}$ and during that period of time the system voltage drops by $20 \%$. 

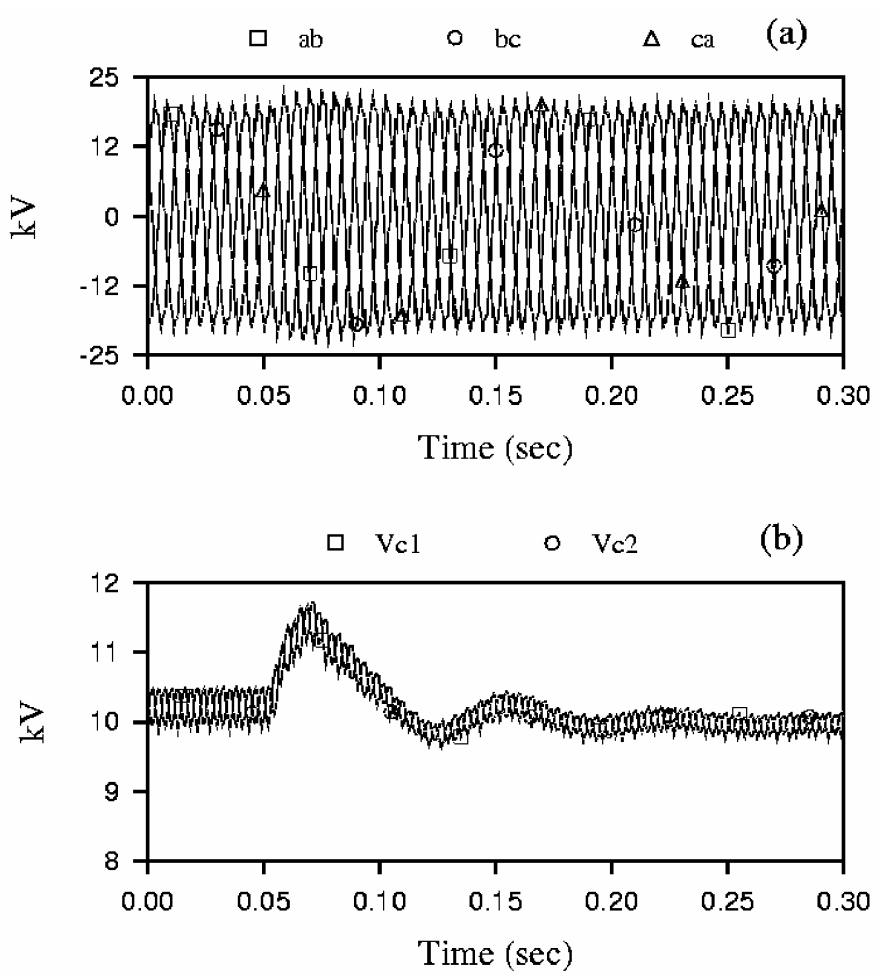

Figure 12. Load rejection waveforms.
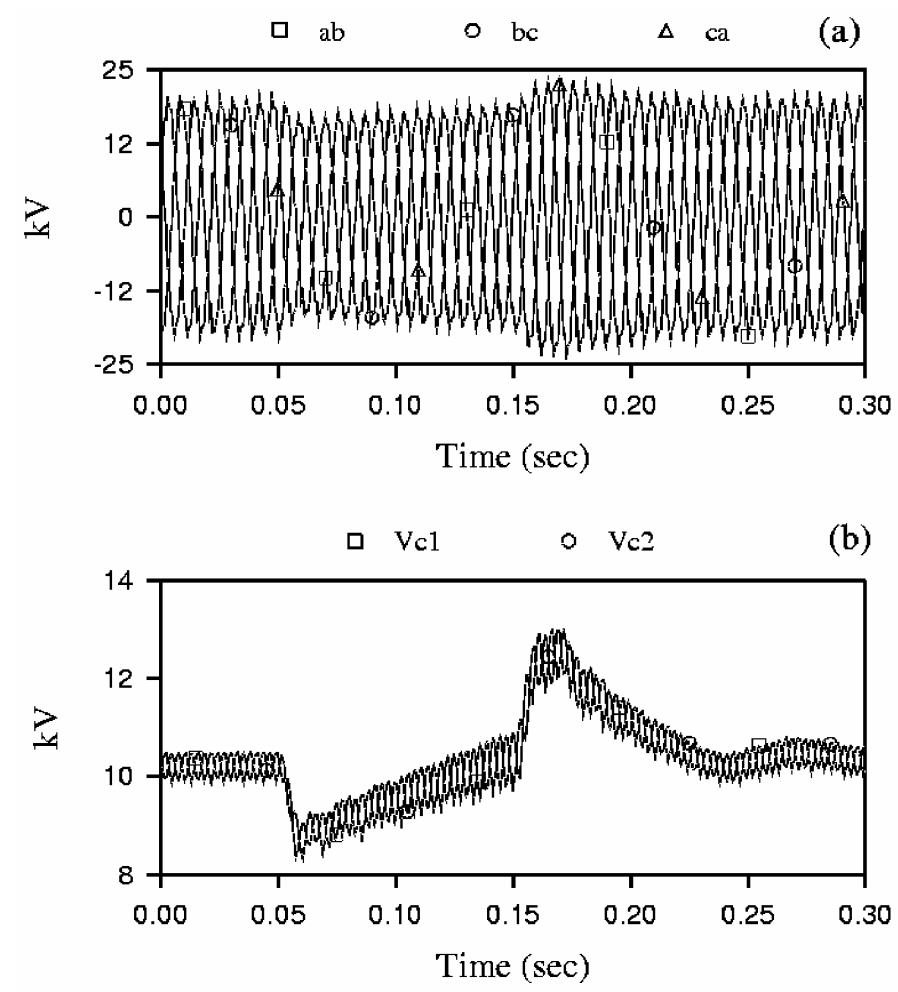

Figure 13. Internal fault waveforms. 
The results of this study are shown in Figure 13. At first we observe a 15\% voltage drop on the bus as Figure 13(a) clearly illustrates. This happens at the onset of the fault. Having reached the maximum point of its capacity however, the compensator can restore the bus voltage to only $95 \%$ of its steady state value.

The restoration of the steady state condition before the disturbance is accomplished in about 4 cycles after the clearing of the fault, indicating the fast response and effectiveness of the compensator. During the fault, the equalization control maintains the capacitors equally charged, as seen on Figure 13(b). The dc control loop reacts rapidly and tries to charge the two capacitors after the initial sudden change. When the fault has been cleared, the dc control discharges the capacitors to a value equal to that of the steady state.

\section{Conclusion}

The case studies presented in this paper required the utilization of two professional grade software packages, the MATLAB control toolbox and the PSCAD/EMTDC real-time simulation software. Both packages have proven to be invaluable tools in the training of the students who enroll in the EIET program, and they are used by professionals and utility companies nationwide. Hence the students are exposed to the operation of tools that most likely they will have to use after they join the workforce.

The authors believe that modern teaching facilities supported with digital simulation tools and well equipped laboratories, have a great impact in the development of engineering technology programs in power systems and energy technologies. A good example of this perception is the fact that the EIET program at UNI has recently completed its first year of operation with great success and increased enrollment. Part of this achievement was due to the fact that the faculty uses advanced software and hardware tools to educate the students that choose the EIET major. This in turn allows for the continuous improvement of the curriculum so that it is always modern and attached to the trends of the ever-expanding electrical engineering technology.

References

1. G.T. Heydt \& V. Vittal, Feeding Our Profession, IEEE Power \& Energy Magazine, 1/2003.

2. MATLAB Student Version, Release 11 Manual, The MathWorks Inc., 1999.

3. PSCAD/EMTDC User's Manual, Manitoba HVDC Research Center, Canada, 2001.

4. C. Johnson \& H. Malki, Control Systems Technology, New Jersey, Prentice Hall, 2002.

5. D. Hanselman \& B. Littlefield, Mastering MATLAB 6, New Jersey, Prentice Hall, 2001.

6. N. Hingorani, L. Gyugyi, Understanding Facts, New Jersey, IEEE Press, 2000.

7. C.J. Hatziadoniu \& F. Chalkiadakis, "A Power Conditioner for a Grid-connected Photovoltaic Generator Based on the 3-level Inverter", IEEE Trans. on Energy Conversion, Vol. 14, No 4, December 1999, pp. 1605-1610.

8. C.J. Hatziadoniu \& F. Chalkiadakis, "A Transformerless High-pulse Static Synchronous Compensator Based on the 3-level GTO Inverter", IEEE Trans. on Power Delivery, Vol. 13, No. 3, July 1998, pp. 883-888. 
Biographies

FANOURIOS (FANIS) CHALKIADAKIS

Fanis Chalkiadakis received his Ph.D. degree in Engineering Science in 2001, from Southern Illinois University at Carbondale. He is an Assistant Professor with the Dept. of Industrial Technology at the University of Northern Iowa and holds memberships in the IEEE Power/Magnetic Societies and the NAIT. His interests include power systems, modeling, circuit theory, microcontrollers, analog electronics, control systems, and laboratory development.

\section{RECAYI PECEN}

Recayi Pecen received his Ph.D. degree in Electrical Engineering from the University of Wyoming in 1997. He is an Assistant Professor at the University of Northern Iowa, Department of Industrial Technology. His research interests include AC/DC power systems, power systems control, power systems harmonics, power quality and renewable energy applications. Dr. Pecen is a member of IEEE, ASEE, NAIT, and Tau Beta Pi Engineering Honor Society. 